\title{
All-optical helicity-dependent magnetic switching by first-order azimuthally polarized vortex beams
}

Cite as: Appl. Phys. Lett. 113, 171108 (2018); https://doi.org/10.1063/1.5051576

Submitted: 10 August 2018 . Accepted: 09 October 2018. Published Online: 24 October 2018

Sicong Wang, Chen Wei, Yuanhua Feng, Yaoyu Cao, Haiwei Wang (D), Weiming Cheng, Changsheng Xie, Arata Tsukamoto, Andrei Kirilyuk (D), Theo Rasing, Alexey V. Kimel, and Xiangping Li (D)

\section{ARTICLES YOU MAY BE INTERESTED IN}

Towards massively parallelized all-optical magnetic recording

Journal of Applied Physics 123, 213904 (2018); https://doi.org/10.1063/1.5003713

Single shot ultrafast all optical magnetization switching of ferromagnetic Co/Pt multilayers Applied Physics Letters 111, 042401 (2017); https://doi.org/10.1063/1.4994802

Perspective: Ultrafast magnetism and THz spintronics

Journal of Applied Physics 120, 140901 (2016); https://doi.org/10.1063/1.4958846

\section{Applied Physics Letters}

Mid-IR and THz frequency combs special collection

Read Now! 


\title{
All-optical helicity-dependent magnetic switching by first-order azimuthally polarized vortex beams
}

\author{
Sicong Wang, ${ }^{1,2}$ Chen Wei, ${ }^{1}$ Yuanhua Feng, ${ }^{3}$ Yaoyu Cao, ${ }^{1}$ Haiwei Wang, ${ }^{4}$ Weiming Cheng,${ }^{5}$ \\ Changsheng Xie, ${ }^{4}$ Arata Tsukamoto, ${ }^{6}$ Andrei Kirilyuk, ${ }^{2,7}$ Theo Rasing, ${ }^{2}$ Alexey V. Kimel, ${ }^{2}$ \\ and Xiangping $\mathrm{Li}^{1, \mathrm{a})}$ \\ ${ }^{1}$ Guangdong Provincial Key Laboratory of Optical Fiber Sensing and Communications, Institute of Photonics \\ Technology, Jinan University, Guangzhou 510632, China \\ ${ }^{2}$ Radboud University, Institute for Molecules and Materials, Heyendaalseweg 135, 6525 AJ Nijmegen, \\ The Netherlands \\ ${ }^{3}$ Department of Electronic Engineering, College of Information Science and Technology, Jinan University, \\ Guangzhou 510632, China \\ ${ }^{4}$ Wuhan National Laboratory for Optoelectronics, Huazhong University of Science and Technology, \\ Wuhan 430074, China \\ ${ }^{5}$ School of Optical and Electronic Information, Huazhong University of Science and Technology, \\ Wuhan 430074, China \\ ${ }^{6}$ College of Science and Technology, Nihon University, 7-24-1 Funabashi, Chiba 274-8501, Japan \\ ${ }^{7}$ Radboud University, FELIX Laboratory, Institute for Molecules and Materials, Toernooiveld 7c, 6525 ED \\ Nijmegen, The Netherlands
}

(Received 10 August 2018; accepted 9 October 2018; published online 24 October 2018)

\begin{abstract}
In this letter, a method to realize all-optical helicity-dependent magnetic switching (AO-HDS) using a first-order azimuthally polarized vortex (FAPV) beam is demonstrated. Numerical calculations of the focal fields of FAPV beams reveal that left-handed and right-handed circular polarizations are generated due to the interaction between the polarization singularity and the helical wave front. Its feasibility for AO-HDS is experimentally demonstrated in $\mathrm{Gd}_{27} \mathrm{Fe}_{63.87} \mathrm{Co}_{9.13}$ under low numerical aperture (NA) conditions and within a narrow fluence window. It is numerically predicted that under high NA conditions, the lateral size of magnetic bits recorded by FAPV beams can be nearly $30 \%$ smaller than that obtained by circularly polarized beams, which opens a promising route to realize ultrafast and ultrahigh-density magnetic recording. Published by AIP Publishing.
\end{abstract}

https://doi.org/10.1063/1.5051576

The research upsurge into ultrafast magnetization dynamics was triggered by the observation of sub-picosecond demagnetization dynamics in nickel thin films after $60 \mathrm{fs}$ pulsed-laser excitation ${ }^{1}$ and the subsequent discovery of alloptical helicity-dependent magnetic switching (AO-HDS) in ferrimagnetic $\mathrm{GdFeCo}$ by circularly polarized (CP) light. ${ }^{2}$ Initially, it was assumed that magnetization switching could only be accomplished with circular polarization. Later, purely thermal switching by linearly polarized light was demonstrated $^{3,4}$ and a lot of research into several hypotheses, including helicity-dependent effective magnetic fields via the inverse Faraday effect (IFE), ${ }^{2,5,6}$ magnetic circular dichroism $(\mathrm{MCD}){ }^{7}$ and exchange coupling between the rare earth (RE) and transition metal (TM) sub-lattices ${ }^{3}$ was done in an attempt to explain the observed helicity-dependent and helicityindependent phenomena. Among these opto-magnetic phenomena, AO-HDS with deterministic direction information is of interest for applications in modern magnetic storage as well as spintronics.

Usually, AO-HDS in ferrimagnetic GdFeCo occurs within a narrow window of pulse energies of the incident light. No switching and helicity-independent switching is achieved below and above this window, respectively. AO-HDS has also been demonstrated in other ferrimagnetic materials $\left(\mathrm{TbCo}^{8}{ }^{8} \mathrm{TbFe},{ }^{9-12} \mathrm{DyCo},{ }^{13} \mathrm{HoFeCo},{ }^{13}\right.$ etc.),

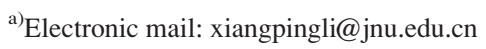

engineered RE/TM multilayers, RE-TM heterostructures, ${ }^{13}$ and even in ferromagnetic materials $(\mathrm{Co} / \mathrm{Pt}$ multilayers, FePtAgC granular films, etc.). ${ }^{14}$ In summary, helical electromagnetic fields of incident femtosecond pulses play a significant role in light-matter interactions and the subsequent AO-HDS in a wide variety of opto-magnetic materials. ${ }^{15-17}$

In addition to helical polarization distributions, helical wave fronts or phase distributions carried by vortex beams are of paramount importance to light-matter interactions as well. ${ }^{18-21}$ It has been shown that circular polarization with high purity within the focal region can be generated by focusing first-order azimuthally polarized vortex (FAPV) beams, due to the interaction between the incident polarization singularity and the helical wave front of light. ${ }^{22}$ Its combination with opto-magnetic control has led to purely longitudinal magnetization needles ${ }^{22}$ and spherical magnetization chains ${ }^{23,24}$ with diffraction-limited feature sizes via the IFE. Furthermore, the focal spot size of FAPV beams can be smaller than that of $\mathrm{CP}$ beams under high numerical aperture (NA) conditions, ${ }^{25,26}$ which holds great potential for high density AO-HDS. However, although a number of promising prospects of FAPV beams have been theoretically predicted, their practical implementations with opto-magnetic materials are yet to be demonstrated.

In this letter, we experimentally demonstrate that under low NA conditions, FAPV beams can reproducibly reverse the magnetization direction in ferrimagnetic $\mathrm{Gd}_{27} \mathrm{Fe}_{63.87} \mathrm{Co}_{9.13}$ in a 
helicity-dependent manner. A window of pulse energies of about $1.8 \%$ for the AO-HDS is revealed. It is numerically shown that under high NA conditions, this AO-HDS process promises nearly $30 \%$ smaller recorded magnetic bits compared with those achieved by ordinary $\mathrm{CP}$ beams.

According to the Debye diffraction theory, ${ }^{27-30}$ the focal electric fields of FAPV beams can be expressed as

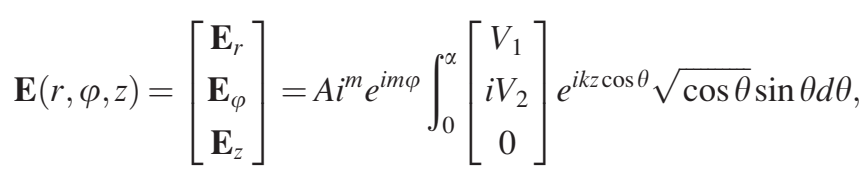

where

$$
\begin{aligned}
& V_{1}=J_{m-1}(k r \sin \theta)+J_{m+1}(k r \sin \theta), \\
& V_{2}=J_{m-1}(k r \sin \theta)-J_{m+1}(k r \sin \theta) .
\end{aligned}
$$

Here, $A$ is a constant and $\mathrm{m}$, which equals \pm 1 , is the topological charge of the helical phase. $r, \varphi, z$, and $k$ are the cylindrical coordinates and the value of the wave vector in the focal space, respectively. $J_{\mathrm{m}-1}$ and $J_{\mathrm{m}+1}$ denote Bessel functions of the first kind. $\alpha$ is the converging angle corresponding to the radius of the incident beam. Equation (1) is represented by cylindrical vector components and indicates that the incident purely azimuthal polarization is transformed into radial and azimuthal polarization components in the focal region due to the mutual interaction between the incident polarization singularity and the helical phase of light. Moreover, a phase difference of $\pi / 2$ exists between the radial and azimuthal components, which means that circular or elliptical polarizations are generated in the focal region.

Figure 1(a) shows the calculated normalized electricfield distributions in the focal plane. The wavelength is
$400 \mathrm{~nm}$ and the NA equals 0.005 . Left-handed and righthanded circular polarizations are generated by focusing FAPV beams with $\mathrm{m}=1$ and $\mathrm{m}=-1$, respectively. In order to reduce the lateral size of the focal spot as much as possible, annular incident beams ${ }^{31}$ are commonly introduced besides increasing the NA of the focal objective. Figure 1(b) shows the dependence of the lateral size of the focal spots on the NA and the annular factor $(\beta)$ of the incident FAPV beams, where $\beta$ is defined as the ratio of the inner and outer radii of the incident annular beam. In Fig. 1(b), the upper and the lower surfaces represent the full width at half maximum (FWHM) of the focal spots of CP and FAPV beams, respectively. It can be seen that under high NA conditions (NA > 0.7), ${ }^{27}$ the focal spot of the FAPV beam is smaller than that formed by the $\mathrm{CP}$ beam and moreover this tendency turns more pronounced with higher NA and larger values of $\beta$. Figure 1(c) shows the focal size reduction versus NA and $\beta$ by focusing FAPV beams compared to $\mathrm{CP}$ beams. Specifically, when NA $=0.95$ and $\beta=0.95$, the lateral focal sizes of the CP and the FAPV beams are $228 \mathrm{~nm}$ and $160 \mathrm{~nm}$, respectively, which corresponds to a focal size reduction of nearly 30\%, making FAPV beams interesting for nanoscale magnetic switching.

Figure 1(d) shows the schematic setup to demonstrate this helicity-dependent prediction. We used $\mathrm{GdFeCo}$, which was well studied in AO-HDS, in our experiments. The amorphous ferrimagnetic $\mathrm{Gd}_{27} \mathrm{Fe}_{63.87} \mathrm{Co}_{9.13}$ sample was grown by magnetron sputtering in a multilayer structure: glass/AlTi $(10 \mathrm{~nm}) / \mathrm{SiN}(5 \mathrm{~nm}) / \mathrm{GdFeCo}(20 \mathrm{~nm}) / \mathrm{SiN}(60 \mathrm{~nm})$, where the AlTi layer served as a heat sink and $\mathrm{SiN}$ was used as a buffer and capping layer. Thin films of this alloy usually exhibit strong perpendicular magnetic anisotropy, and have a Curie point $T_{\mathrm{C}} \approx 550 \mathrm{~K}$, a magnetization compensation point $T_{\mathrm{M}}$ $\approx 474.5 \mathrm{~K}$, and an angular momentum compensation point (a)
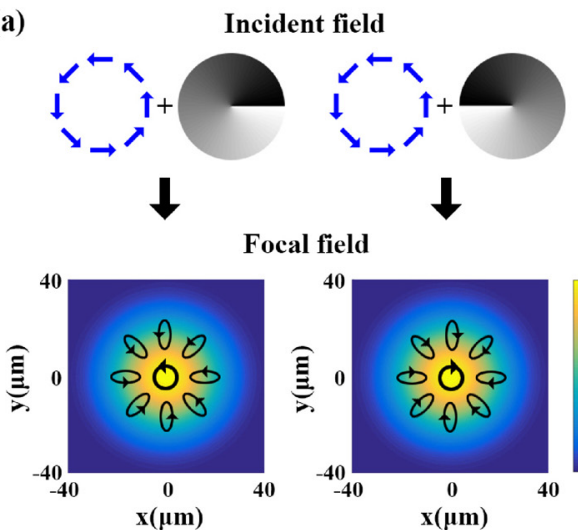

Focal field

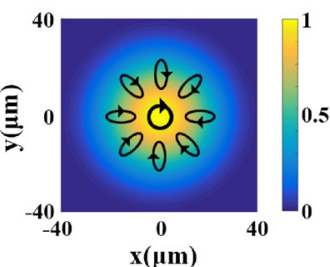

(b)

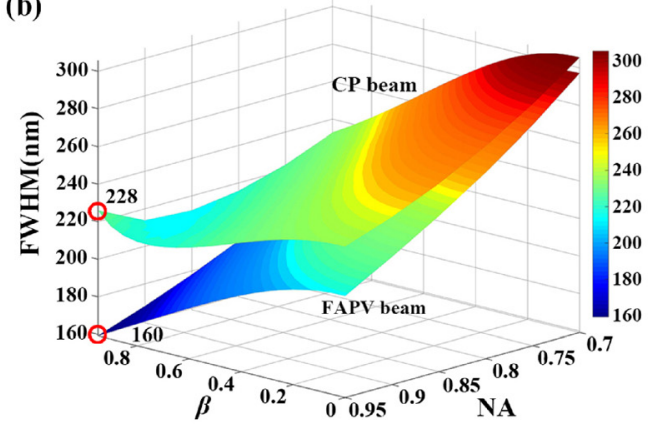

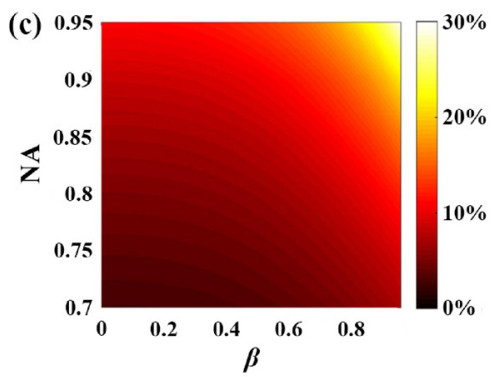

(d)

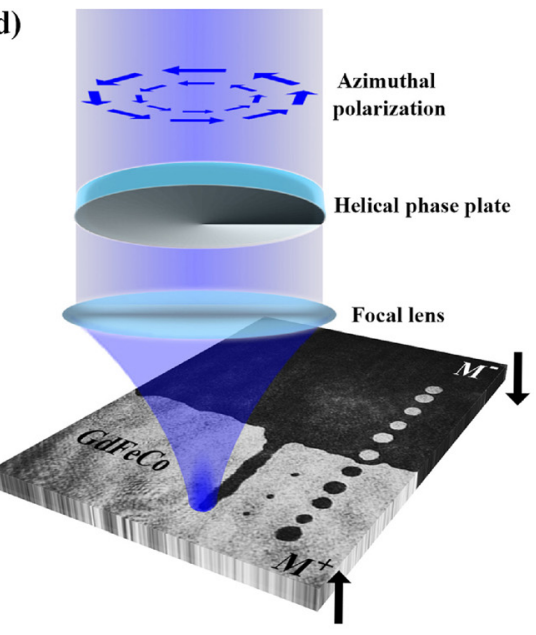

FIG. 1. (a) Calculated normalized electric-field distributions in the focal plane by focusing FAPV beams with a wavelength of $400 \mathrm{~nm}(\mathrm{NA}=0.005)$. Left-handed and right-handed circular polarizations are generated in the focal region when $\mathrm{m}=1$ and $\mathrm{m}=-1$, respectively. (b) Dependence of the lateral focal size on the NA and the annular factor $\beta$. The upper and the lower surfaces represent the FWHMs of the focal spots of CP and FAPV incident beams, respectively. (c) The focal size reduction versus NA and $\beta$ by focusing FAPV beams compared with the focal size achieved by CP beams. (d) Schematic illustration of the setup to realize AO-HDS by focusing a FAPV beam. 
$T_{\mathrm{A}} \approx 524.5 \mathrm{~K}^{32-34}$ The magnetic domain structures were imaged through the magneto-optical Faraday effect of a linearly polarized pulsed beam from an amplified Ti:sapphire laser. The repetition rate, pulse width, and the central wavelength of the imaging laser pulses were $1 \mathrm{kHz}, 40 \mathrm{fs}$, and $800 \mathrm{~nm}$, respectively. Magnetic domains with magnetization parallel ("up") or antiparallel ("down") to the sample normal are shown as white or black regions, respectively, in the image of a CCD camera. The sample was excited by a single laser pulse or sweeping a pulse train with a central wavelength of $400 \mathrm{~nm}$, which was obtained through frequency doubling of the regeneratively amplified $800 \mathrm{~nm}$ pulses by a $\mathrm{BBO}$ crystal. A pulse picker was used to reduce the repetition rate of the excitation pulses. The polarization of the excitation pulses was converted to azimuthal polarization by an ARCoptix polarization converter. A helical phase plate with topological charges of \pm 1 , namely a helical $0-2 \pi$ phase plate, was utilized to generate the FAPV beam. The experiments were performed at room temperature in air.

To verify the feasibility of AO-HDS in $\mathrm{Gd}_{27} \mathrm{Fe}_{63.87} \mathrm{Co}_{9.13}$ by FAPV beams without ambiguity, we performed experiments with a low NA focal lens $(\mathrm{NA}=0.005)$ which focused the incident laser beam down to a spot with a FWHM of around $42 \mu \mathrm{m}$. Figure 2(a) shows the initial magnetization structure before the excitation of the incident light. The scale bar is $50 \mu \mathrm{m}$. The results achieved by the excitation of the FAPV beams with $\mathrm{m}=1$ and $\mathrm{m}=-1$ are shown in Figs. 2(b) and 2(c), respectively. In Figs. 2(b) and 2(c), the dots were written by single laser pulses with a fluence of $5.08 \mathrm{~mJ} / \mathrm{cm}^{2}$ on the sample. The fluence is defined as the pulse energy divided

(a)

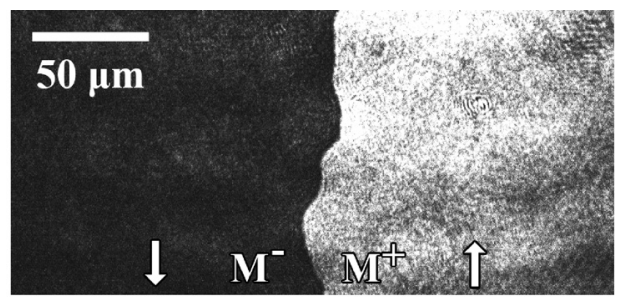

(b)

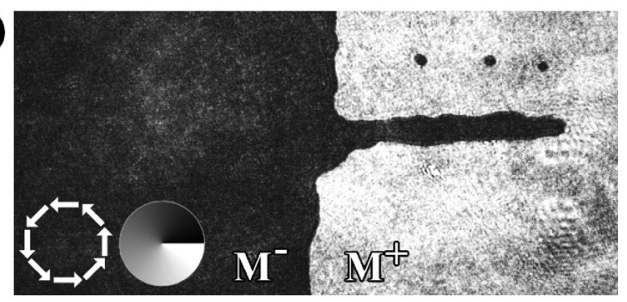

(c)

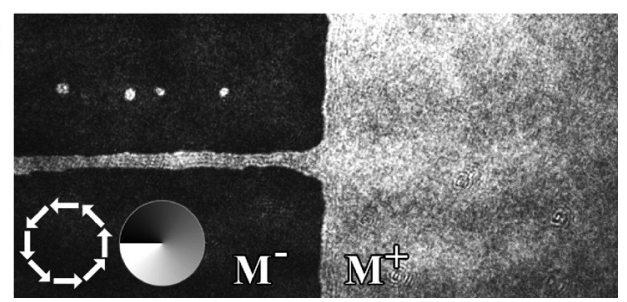

FIG. 2. (a) The initial magnetization structure before light excitation. The white and black regions represent magnetization distributions with orientations "up" $\left(\mathrm{M}^{+}\right)$and "down" $\left(\mathrm{M}^{-}\right)$, respectively. (b) The results achieved by the excitation of a FAPV beam with $\mathrm{m}=1$. The dots are generated by single laser pulses and the line structure is formed through sweeping an incident pulse train with a repetition rate of $100 \mathrm{~Hz}$. (c) The results achieved by the excitation of a FAPV beam with $\mathrm{m}=-1$. (a)
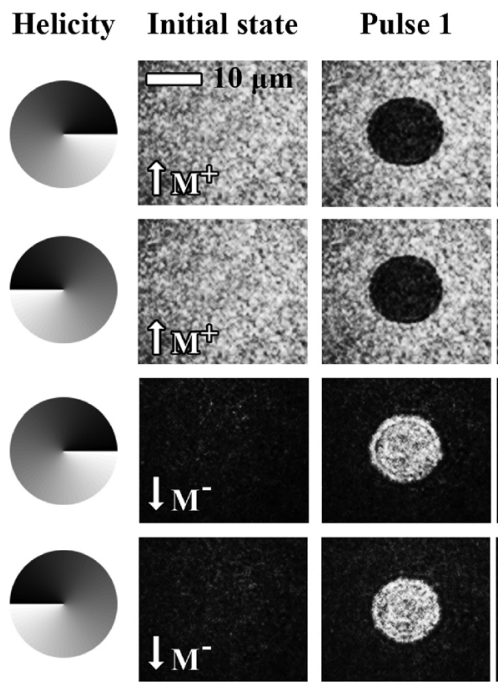

(b)

(c)

FIG. 3. Helicity-dependent and helicity-independent switching formed by laser pulses with different fluences. (a) Combinations between the helicity of the incident FAPV beam and the initial state of the magnetization. The scale bar is $10 \mu \mathrm{m}$. (b) Helicity-independent switching generated by two consecutive laser pulses with a fluence of $5.51 \mathrm{~mJ} / \mathrm{cm}^{2}$. Annular switching patterns are finally formed in the regions where the conditions of AO-HDS are satisfied. (c) Helicity-dependent switching formed by a single laser pulse with a fluence of $5.08 \mathrm{~mJ} / \mathrm{cm}^{2}$.

by the beam area which is measured through the Liu method. ${ }^{35}$ It can be seen that AO-HDS occurs under this light fluence. In the case of $m=1$, small black domains are recorded in the white region while nothing occurs in the black region. The opposite happens when $m=-1$. This AO-HDS is further demonstrated by sweeping the FAPV pulsed beams with a repetition rate of $100 \mathrm{~Hz}$ across the domain wall between the white and black regions. The sweeping speed of the beam was around $29.8 \mu \mathrm{m} / \mathrm{s}$. Similar to the case of single pulse excitation, helicity-dependent line structures are formed in the corresponding regions. In contrast, with a higher light fluence, helicity-independent switching occurs. Figure 3(a) shows the

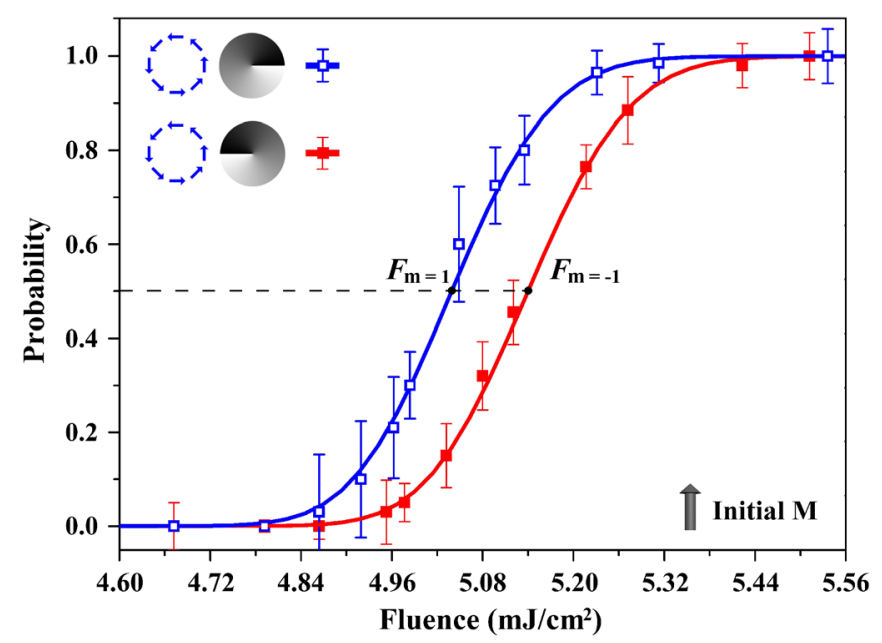

FIG. 4. Switching probability as a function of the fluence of the incident FAPV single pulses with different topological charges. The blue hollow and the red solid squares with error bars represent the switching probabilities under the excitation of the FAPV single pulses with $\mathrm{m}=1$ and $\mathrm{m}=-1$, respectively. The data are fitted with an error function. The magnetization orients "up" initially. 


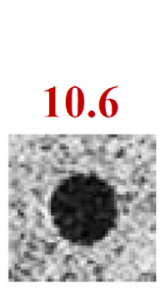

5.40

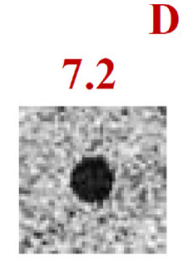

5.25

Diameter $(\mu \mathrm{m})$

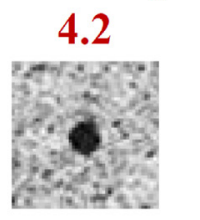

Fluence $\left(\mathrm{mJ} / \mathrm{cm}^{2}\right)$
5.10 $\mathrm{m}^{2}$

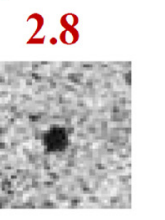

4.95

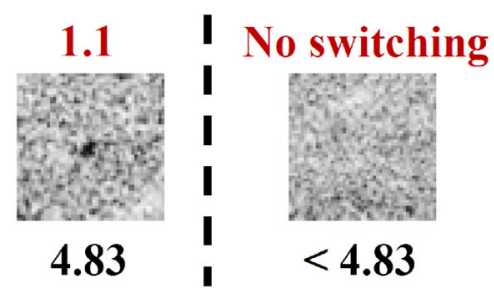

FIG. 5. Switching areas obtained under the excitation of FAPV beams $(m=1)$ with different fluences. The minimum switching diameter is around $1.1 \mu \mathrm{m}$ at a fluence of $4.83 \mathrm{~mJ} / \mathrm{cm}^{2}$. No switching is observed when the fluence is lower than $4.83 \mathrm{~mJ} / \mathrm{cm}^{2}$. combinations between the helicity of the incident FAPV beam and the initial state of the magnetization. As shown in Fig. 3(b), under the excitation of two consecutive laser pulses with a fluence of $5.51 \mathrm{~mJ} / \mathrm{cm}^{2}$, magnetic switching happens regardless of the helicity of the imposed phase or the initial state of the magnetization. Notably, as the light fluence at the rim of the switching region is lower than that in the center, annular switching patterns are finally formed in the regions where the conditions of AO-HDS are satisfied. Figure 3(c) shows the AO-HDS generated by a single laser pulse with a fluence of $5.08 \mathrm{~mJ} / \mathrm{cm}^{2}$. These phenomena occur from the fact that alloptical magnetic switching in GdFeCo alloys dominantly stems from the exchange interaction between the $\mathrm{Gd}$ and $\mathrm{Fe}$ sublattices via a transient ferromagnetic-like sate owing to the substantially different opto-magnetic dynamics of the $\mathrm{Gd}$ and Fe sublattices. ${ }^{3,4}$ Furthermore, AO-HDS occurs only in an appropriate fluence range of the exciting pulses, which is ascribed to the MCD. ${ }^{7}$ Above this range, helicity-independent switching would occur since both the left-handed and righthanded circularly polarized light can exceed the threshold switching fluence.

The switching probability as a function of the fluence is shown in Fig. 4. The initial magnetization was in the "up" direction. This procedure was repeated about 20 times at every pulse energy. The blue hollow and the red solid squares with error bars represent the switching probabilities under the excitation of the FAPV single pulses with $\mathrm{m}=1$ and $m=-1$, respectively. The experimental data are fitted with an error function. ${ }^{7}$ It can be seen that a distinct fluence window $(\Delta)$ of AO-HDS of around $1.8 \%$ is observed, which is calculated by

$$
\Delta=\frac{F_{m=-1}-F_{m=1}}{\frac{1}{2}\left(F_{m=-1}+F_{m=1}\right)},
$$

where $F_{\mathrm{m}=1}$ and $F_{\mathrm{m}=-1}$ are defined as the threshold switching fluences of $\mathrm{Gd}_{27} \mathrm{Fe}_{63.87} \mathrm{Co}_{9.13}$ excited by the FAPV beams with $\mathrm{m}=1$ and $\mathrm{m}=-1$, respectively. The switching probability is 0.5 at the corresponding threshold fluence. This narrow window induced by the MCD indicates that AO-HDS occurs in an appropriate fluence range of the exciting pulses. Beyond this window, no switching or helicityindependent switching occurs, which is similar to the previous results achieved by purely $\mathrm{CP}$ beams. ${ }^{7}$ The slope of the probability curves originates from pulse to pulse intensity fluctuations.

So far, AO-HDS excited by the FAPV beams has been experimentally demonstrated in $\mathrm{Gd}_{27} \mathrm{Fe}_{63.87} \mathrm{Co}_{9.13}$ under both single-pulse and multi-pulse conditions. The switching areas obtained under the excitation of FAPV beams $(\mathrm{m}=1)$ with different fluences are shown in Fig. 5. It can be seen that the switched area turns smaller with lower excitation fluence of the FAPV beam. However, the smallest switching diameter obtained in our experiment was limited to around $1.1 \mu \mathrm{m}$ at a fluence of $4.83 \mathrm{~mJ} / \mathrm{cm}^{2}$, even by using focal lenses with increased NA. No switching was observed when the fluence was lower than $4.83 \mathrm{~mJ} / \mathrm{cm}^{2}$. This relatively large switching diameter is due to the fact that the GdFeCo films are magnetically soft, limiting the smallest achievable domain sizes to about $1-3 \mu \mathrm{m} .{ }^{36}$ Although GdFeCo is therefore not optimal for demonstrating magnetic recording on the nanoscale, these results can inspire future developments to apply FAPV beams to other ferrimagnets (such as TbFe, $\mathrm{TbCo}$, and $\mathrm{TbFeCo})^{8-12,37}$ and ferromagnets $(\mathrm{Co} / \mathrm{Pt}$ multilayers, FePtAgC granular films, etc. $)^{14}$ which possess stable nanoscale magnetic domains.

In conclusion, in our work, FAPV beams are introduced to realize AO-HDS. By reversing the wave front helicity of the FAPV beams, deterministic AO-HDS is unambiguously observed in $\mathrm{Gd}_{27} \mathrm{Fe}_{63.87} \mathrm{Co}_{9.13}$ under low NA conditions. A fluence window of about $1.8 \%$ for AO-HDS is obtained. Numerical calculations predict that compared with $\mathrm{CP}$ beams, a switching size reduction of nearly $30 \%$ can be achieved by using FAPV beams under high NA conditions, which enables magnetic switching of nanoscale domains. Even though the large domain size of the magnetically soft $\mathrm{GdFeCo}$ films restricts the demonstration of nanoscale magnetic switching in this material, ferromagnets and other ferrimagnets with nanoscale domain sizes may take full advantage of FAPV beams and be applied to realize nanoscale magnetic switching. The results in our work open a promising route to realize ultrahigh-density AO-HDS and may inspire subsequent developments in closely related fields, including data storage and spintronics.

We are grateful to Dr. Dmytro V. Afanasiev, Tonnie Toonen, Dr. Bowen Jiang, Dr. Sergey Semin, and Jianjun Luo for their professional technical support. This letter was financially supported by the National Key R\&D Program of China (YS2018YFB110012), the National Natural Science Foundation of China (NSFC) (11604123, 61522504, 61432007, 61605065, and 61475038), the Fundamental Research Funds for the Central Universities (21616338), the Guangdong Provincial Innovation and Entrepreneurship Project (Grant No. 2016ZT06D081), the Grant-in-Aid for Scientific Research on Innovative Area, "Nano Spin Conversion Science" (Grant No. 26103005), the European Research Council ERC, Grant Agreement No. 339813 
(Exchange), and the Netherlands Organization for Scientific Research (NWO).

${ }^{1}$ E. Beaurepaire, J.-C. Merle, A. Daunois, and J.-Y. Bigot, Phys. Rev. Lett. 76, 4250 (1996).

${ }^{2}$ C. D. Stanciu, F. Hansteen, A. V. Kimel, A. Kirilyuk, A. Tsukamoto, A. Itoh, and T. Rasing, Phys. Rev. Lett. 99, 047601 (2007).

${ }^{3}$ I. Radu, K. Vahplar, C. Stamm, T. Kachel, N. Pontius, H. A. Dürr, T. A. Ostler, J. Barker, R. F. L. Evans, R. W. Chantrell, A. Tsukamoto, A. Itoh, A. Kirilyuk, T. Rasing, and A. V. Kimel, Nature 472, 205 (2011).

${ }^{4}$ T. A. Ostler, J. Barker, R. F. L. Evans, R. W. Chantrell, U. Atxitia, O. Chubykalo-Fesenko, S. El Moussaoui, L. Le Guyader, E. Mengotti, L. J. Heyderman, F. Nolting, A. Tsukamoto, A. Itoh, D. Afanasiev, B. A. Ivanov, A. M. Kalashnikova, K. Vahaplar, J. Mentink, A. Kirilyuk, T. Rasing, and A. V. Kimel, Nat. Commun. 3, 666 (2012).

${ }^{5}$ K. Vahaplar, A. M. Kalashnikova, A. V. Kimel, D. Hinzke, U. Nowak, R. Chantrell, A. Tsukamoto, A. Itoh, A. Kirilyuk, and T. Rasing, Phys. Rev. Lett. 103, 117201 (2009).

${ }^{6}$ A. Kirilyuk, A. V. Kimel, and T. Rasing, Rev. Mod. Phys. 82, 2731 (2010).

${ }^{7}$ A. R. Khorsand, M. Savoini, A. Kirilyuk, A. V. Kimel, A. Tsukamoto, A. Itoh, and T. Rasing, Phys. Rev. Lett. 108, 127205 (2012).

${ }^{8}$ S. Alebrand, M. Gottwald, M. Hehn, D. Steil, M. Cinchetti, D. Lacour, E. E. Fullerton, M. Aeschlimann, and S. Mangin, Appl. Phys. Lett. 101, 162408 (2012).

${ }^{9}$ A. Hassdenteufel, B. Hebler, C. Schubert, A. Liebig, M. Teich, M. Helm, M. Aeschlimann, M. Albrecht, and R. Bratschitsch, Adv. Mater. 25, 3122 (2013).

${ }^{10}$ C. Schubert, A. Hassdenteufel, P. Matthes, J. Schmidt, M. Helm, R. Bratschitsch, and M. Albrecht, Appl. Phys. Lett. 104, 082406 (2014).

${ }^{11}$ A. Hassdenteufel, C. Schubert, J. Schmidt, P. Richter, D. R. T. Zahn, G. Salvan, M. Helm, R. Bratschitsch, and M. Albrecht, Appl. Phys. Lett. 105, 112403 (2014).

${ }^{12}$ A. Hassdenteufel, J. Schmidt, C. Schubert, B. Hebler, M. Helm, M. Albrecht, and R. Bratschitsch, Phys. Rev. B 91, 104431 (2015).

${ }^{13}$ S. Mangin, M. Gottwald, C.-H. Lambert, D. Steil, V. Uhlír, L. Pang, M. Hehn, S. Alebrand, M. Cinchetti, G. Malinowski, Y. Fainman, M. Aeschlimann, and E. E. Fullerton, Nat. Mater. 13, 286 (2014).
${ }^{14}$ C.-H. Lambert, S. Mangin, B. S. D. Ch, S. Varaprasad, Y. K. Takahashi, M. Hehn, M. Cinchetti, G. Malinowski, K. Hono, Y. Fainman, M. Aeschlimann, and E. E. Fullerton, Science 345, 1337 (2014).

${ }^{15}$ B. Koopmans, NPG Asia Mater. 6, e146 (2014).

${ }^{16}$ S. Wang, X. Li, J. Zhou, and M. Gu, Opt. Express 23, 13530 (2015).

${ }^{17}$ S. Wang, Y. Cao, and X. Li, Opt. Lett. 42, 5050 (2017).

${ }^{18}$ Z.-L. Deng, J. Deng, X. Zhuang, S. Wang, K. Li, Y. Wang, Y. Chi, X. Ye, J. Xu, G. P. Wang, R. Zhao, X. Wang, Y. Cao, X. Cheng, G. Li, and X. Li, Nano Lett. 18, 2885 (2018).

${ }^{19}$ S. Wang, Z.-L. Deng, Y. Cao, D. Hu, Y. Xu, B. Cai, L. Jin, Y. Bao, X. Wang, and X. Li, IEEE Photonics J. 10, 1 (2018).

${ }^{20}$ S. Wang, X. Ouyang, Z. Feng, Y. Cao, M. Gu, and X. Li, Opto-Electron. Adv. 1, 170002 (2018).

${ }^{21}$ Z. Nie, W. Ding, D. Li, X. Zhang, Y. Wang, and Y. Song, Opt. Express 23, 690 (2015).

${ }^{22}$ S. Wang, X. Li, J. Zhou, and M. Gu, Opt. Lett. 39, 5022 (2014).

${ }^{23}$ L. Gong, L. Wang, Z. Zhu, X. Wang, H. Zhao, and B. Gu, Appl. Opt. 55, 5783 (2016).

${ }^{24}$ W. Yan, Z. Nie, X. Zhang, Y. Wang, and Y. Song, Appl. Opt. 56, 1940 (2017).

${ }^{25}$ X. Hao, C. Kuang, T. Wang, and X. Liu, Opt. Lett. 35, 3928 (2010).

${ }^{26}$ X. Li, P. Venugopalan, H. Ren, M. Hong, and M. Gu, Opt. Lett. 39, 5961 (2014).

${ }^{27} \mathrm{M}$. Gu, Advanced Optical Imaging Theory (Springer, 2000).

${ }^{28}$ X. Li, T. H. Lan, C. H. Tien, and M. Gu, Nat. Commun. 3, 998 (2012).

${ }^{29}$ X. Li, Y. Cao, and M. Gu, Opt. Lett. 36, 2510 (2011).

${ }^{30}$ S. Wang, X. Xie, M. Gu, and J. Zhou, J. Opt. Soc. Am. A 32, 1026 (2015).

${ }^{31}$ G. M. Lerman and U. Levy, Opt. Express 16, 4567 (2008).

${ }^{32}$ Y. Mimura, N. Imamura, T. Kobayashi, A. Okada, and Y. Kushiro, J. Appl. Phys. 49, 1208 (1978).

${ }^{33}$ C. D. Stanciu, A. V. Kimel, F. Hansteen, A. Tsukamoto, A. Itoh, A. Kirilyuk, and T. Rasing, Phys. Rev. B 73, 220402 (2006).

${ }^{34}$ K. Vahaplar, A. M. Kalashnikova, A. V. Kimel, S. Gerlach, D. Hinzke, U. Nowak, R. Chantrell, A. Tsukamoto, A. Itoh, A. Kirilyuk, and T. Rasing, Phys. Rev. B 85, 104402 (2012).

${ }^{35}$ J. M. Liu, Opt. Lett. 7, 196 (1982).

${ }^{36}$ S. Hosaka, T. Shintani, M. Miyamoto, A. Kikukawa, A. Hirotsune, M. Terao, M. Yoshida, K. Fujita, and S. Kämmer, J. Appl. Phys. 79, 8082 (1996).

${ }^{37}$ M. Finazzi, M. Savoini, A. R. Khorsand, A. Tsukamoto, A. Itoh, L. Duo, A. Kirilyuk, T. Rasing, and M. Ezawa, Phys. Rev. Lett. 110, 177205 (2013). 